\title{
Special Issue on Massive MIMO
}

Thomas L. Marzetta, Giuseppe Caire, Mérouane Debbah, Chih-Lin I, and Saif K. Mohammed

Demand for wireless communications is projected to grow by more than a factor of forty or more over the next five years. A potential technology for meeting this demand is Massive MIMO (also called Large-Scale Antenna Systems, Large-Scale MIMO, ARGOS, Full-Dimension MIMO, or Hyper-MIMO), a form of multi-user multipleantenna wireless which promises orders-of-magnitude improvements in spectral-efficiency over 4G technology, and accompanying improvements in radiated energy-efficiency. The distinguishing feature of Massive MIMO is that a large number of service-antennas - possibly hundreds or even thousands - work for a significantly smaller number of active autonomous terminals. Upsetting the traditional parity between service antennas and terminals in this manner is a game-changer: The simplest multiplexing pre-coding and de-coding algorithms can be nearly optimal, expensive ultra-linear forty-Watt power amplifiers are replaced by many low-power units, and the favorable action of the law of large numbers can greatly facilitate power-control and resource-allocation.

Massive MIMO is still an emerging field. There are many unanswered theoretical questions and much remains to be done to obtain a reduction to practice. The six papers in this Special Issue are a sampling of the types of problems that are topics of active research. The papers logically fall into three categories: a) Acquisition of Channel State Information, b) Spatial Multiplexing Algorithms, and c) Massive Array Issues and Architectures.

\section{Acquisition of Channel State Information}

The acquisition of channel state information (CSI) is perhaps the central activity of any Massive MIMO system since possession of CSI by the massive array enables selective focusing of power on the down link and selective collection of power on the up link. Much of the Massive MIMO work has adopted the convenient block fading model. "Effects of Channel Aging in Massive MIMO Systems" by Truong et al. makes the more realistic assumption that CSI degrades gradually rather than abruptly: the performance of the Massive MIMO system does degrade with time, but in a graceful manner. TDD operation permits Massive MIMO to be scalable in the number of service-antennas to any desired extent. Nevertheless there remains considerable interest in making Massive MIMO work for FDD systems which predominate in Europe and North America. "A Channel State Information Feedback Method for Massive MIMO-OFDM" by Kudo et al. investigates an FDD scheme that entails down link pilots and digital CSI feedback on the up link.

\section{Spatial Multiplexing Algorithms}

Spatial multiplexing/de-multiplexing algorithms enable every active terminal to have full access to all of the time/frequency resources and are ultimately responsible for the impressive spectral efficiency of which Massive MIMO is capable. Shannon theory tells us that optimal operation on the down link is obtained by dirty-paper 
coding, and on the up link by joint de-coding. Massive MIMO permits the use of vastly simpler multiplexing/demultiplexing algorithms such as zero-forcing and conjugate beam-forming/matched-filtering. "Optimum Number of Users in Zero-Forcing Based Multiuser MIMO Systems with Large Number of Antennas" by Jung et al. utilizes random matrix theory to obtain formulas for predicting the optimum number of terminals to serve when zeroforcing is employed. Most of the Massive MIMO literature concerns concentrated arrays of service antennas. An alternative deployment for Massive MIMO is a distributed one, in which users over a large area are served simultaneously by a random distribution of access points. "Low Complexity Zero-Forcing Beamforming for Distributed Massive MIMO Systems in Large Public Venues" by Li et al. devises computationally efficient ways of performing zero-forcing for a system of distributed service antennas.

\section{Massive Array Issues and Architectures}

To make Massive MIMO a practical solution it is desirable to develop scalable architectures for massive arrays that are based on inexpensive modular blocks. It is tempting to think that the Law of Large Numbers will compensate for the imperfections of the individual modules. "Widely-Linear Beamforming and RF Impairment Suppression in Massive Antenna Arrays", by Hakkarainen et al. studies the sensitivity of large antenna arrays to RF I/Q imbalance. Additionally, RF aware beamforming methods are proposed to reduce the unwanted effects of RF I/Q imbalance. "Before/After Precoded Massive MIMO in Cloud Radio Access Networks" by Park et al. studies the impact of constraints on backhaul throughputs between the digital unit and the multiplicity of radio units that comprise the massive array. This impacts both the architecture of the array as well as the functionality of the radio units.

In summary, Massive MIMO is a vital, exciting research field as evinced by the papers in this Special Issue, however much remains to be done.

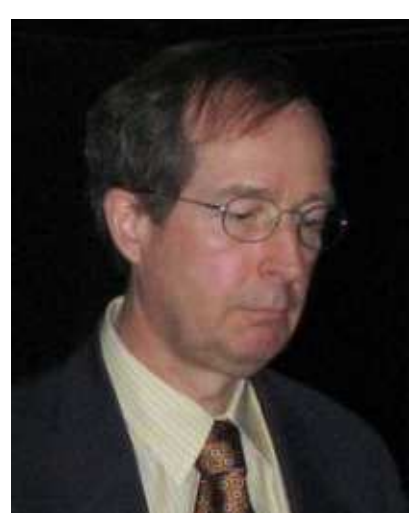

Thomas L. Marzetta was born in Washington, D.C. He received the PhD in Electrical Engineering from the Massachusetts Institute of Technology in 1978. His dissertation extended, to two dimensions, the three-way equivalence of autocorrelation sequences, minimum-phase prediction error filters, and reflection coefficient sequences. He worked for Schlumberger-Doll Research (1978 - 1987) to modernize geophysical signal processing for petroleum exploration. He headed a group at Nichols Research Corporation (1987 - 1995) which improved automatic target recognition, radar signal processing, and video motion detection. He joined Bell Laboratories in 1995 (formerly part of AT\&T, then Lucent Technologies, now Alcatel-Lucent). Within the former Mathematical Sciences Research Center, he was Director of the Communications and Statistical Sciences Department. He was an early proponent of Massive MIMO which can provide huge improvements in wireless spectral-efficiency and energy-efficiency over $4 \mathrm{G}$ technologies. 
He was a Member of the IEEE Signal Processing Society Technical Committee on Multidimensional Signal Processing, a Member of the Sensor Array and Multichannel Technical Committee, an Associate Editor for the IEEE Transactions on Signal Processing, an Associate Editor for the IEEE Transactions on Image Processing, and a Guest Associate Editor for the IEEE Transactions on Information Theory Special Issue on Signal Processing Techniques for Space-Time Coded Transmissions (Oct. 2002), for the IEEE Transactions on Information Theory Special Issue on Space-Time Transmission, Reception, Coding, and Signal Design (Oct. 2003), and for the IEEE JSAC Special Issue on Large-Scale Multiple Antenna Wireless Systems (Feb. 2013). He is currently the Lead Guest Editor for the JCN Special Issue on Massive MIMO (Aug. 2013).

He was the Recipient of the 1981 ASSP Paper Award from the IEEE Signal Processing Society. He was elected a Fellow of the IEEE in Jan. 2003. He received the 2013 IEEE Guglielmo Marconi Best Paper Award.

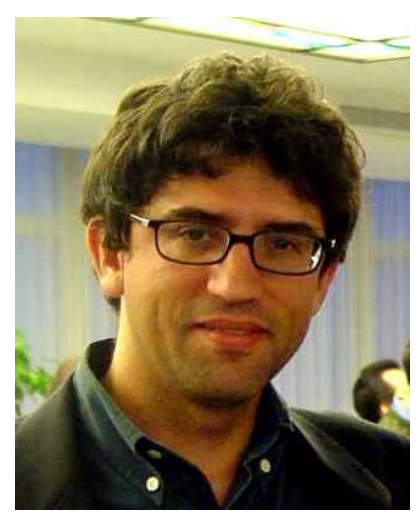

Giuseppe Caire was born in Torino, Italy, in 1965. He received the B.Sc. degree in Electrical Engineering from Politecnico di Torino (Italy), in 1990, the M.Sc. degree in Electrical Engineering from Princeton University in 1992, and the Ph.D. degree from Politecnico di Torino in 1994. He was a Recipient of the AEI G.Someda Scholarship in 1991, has been with the European Space Agency (ESTEC, Noordwijk, The Netherlands) from May 1994 to February 1995, was a Recipient of the COTRAO Scholarship in 1996 and of a CNR Scholarship in 1997.

He has been visiting Princeton University in Summer 1997 and Sydney University in Summer 2000. He has been Assistant Professor in Telecommunications at the Politecnico di Torino, Associate Professor at the University of Parma, Italy, Professor with the Department of Mobile Communications at the Eurecom Institute, Sophia-Antipolis, France, and he is currently a Professor of Electrical Engineering with the Viterbi School of Engineering, University of Southern California, Los Angeles, CA.

He served as Associate Editor for the IEEE Transactions on Communications in 1998-2001 and as Associate Editor for the IEEE Transactions on Information Theory in 2001-2003. He received the Jack Neubauer Best System Paper Award from the IEEE Vehicular Technology Society in 2003, and the IEEE Communications Society \& Information Theory Society Joint Paper Award in 2004 and in 2011. Giuseppe Caire is Fellow of IEEE since 2005. He has served in the Board of Governors of the IEEE Information Theory Society from 2004 to 2007, and as President of the IEEE Information Theory Society in 2011. His main research interests are in the field of communications theory, information theory, channel and source coding with particular focus on wireless communications. 


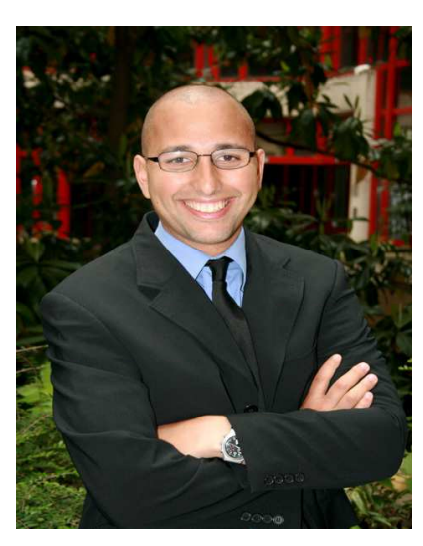

Mérouane Debbah entered the Ecole Normale Supérieure de Cachan (France) in 1996 where he received his M.Sc and Ph.D. degrees, respectively. He worked for Motorola Labs (Saclay, France) from 1999-2002 and the Vienna Research Center for Telecommunications (Vienna, Austria) until 2003. He then joined the Mobile Communications department of the Institut Eurecom (Sophia Antipolis, France) as an Assistant Professor until 2007. He is now a Full Professor at Supelec (Gif-sur-Yvette, France), holder of the Alcatel-Lucent Chair on Flexible Radio and a recipient of the ERC starting grant MORE (Advanced Mathematical Tools for Complex Network Engineering). His research interests are in information theory, signal processing, and wireless communications. He is a Senior Area Editor for IEEE Transactions on Signal Processing and an Associate Editor in Chief of the journal Random Matrix: Theory and Applications. Mérouane Debbah is the recipient of the "Mario Boella" award in 2005, the 2007 General Symposium IEEE GLOBECOM Best Paper Award, the Wi-Opt 2009 Best Paper Award, the 2010 Newcom++ Best Paper Award, the WUN CogCom Best Paper 2012 and 2013 Award as well as the Valuetools 2007, Valuetools 2008, Valuetools 2012 and CrownCom2009 Best Student Paper Awards. He is a WWRF Fellow and an Elected Member of the academic senate of Paris-Saclay. In 2011, he received the IEEE Glavieux Prize Award. He is the Co-Founder of Ximinds.

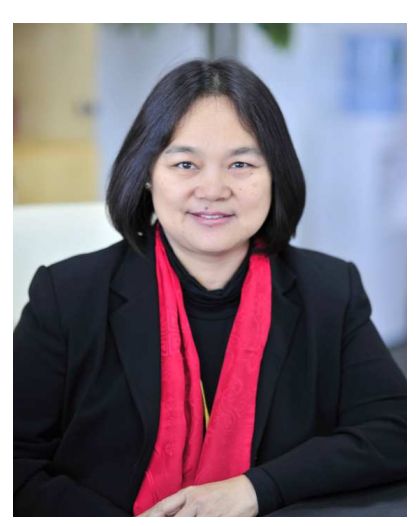

Chih-Lin I, Ph.D. in Electrical Engineering from Stanford University, has almost 30 years experience in wireless communication area. She has worked in various worldclass companies and research institutes, including wireless communication fundamental research department of AT\&T Bell Labs; Headquarter of AT\&T, as Director of Wireless Communications Infrastructure and Access Technology; ITRI of Taiwan, as Director of Wireless Communication Technology Department; Hong Kong ASTRI, as VP and GD of Communications Technology Domain. She was an Elected Board Member of IEEE ComSoc, and the Founding Chair of IEEE WCNC Steering Committee. She is currently an Executive Board Member of GreenTouch, a Winner of CCCP "National 1000 talent" program, China Mobile Chief Scientist of Wireless Technologies, Head of Green Communication Research Center (GCRC) of China Mobile Research Institute (CMRI), in charge of advanced wireless communication R\&D effort of CMRI. 


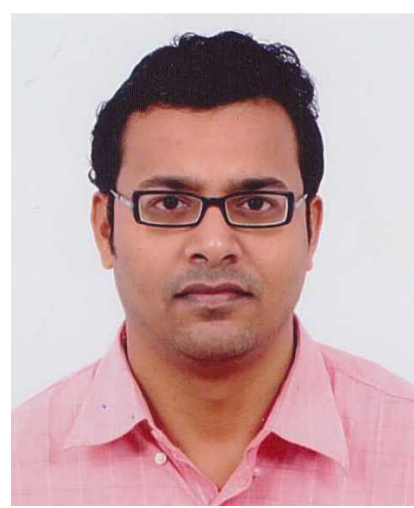

Saif K. Mohammed received the B.Tech degree in Computer Science and Engineering from the Indian Institute of Technology (I.I.T.), New Delhi, India, in 1998 and the Ph.D. degree from the Electrical and Communication Engineering Department, Indian Institute of Science, Bangalore, India, in 2010. Currently, he is an Assistant Professor in the Department of Electrical Engineering, I.I.T. Delhi. From Sept. 2011 to Feb. 2013, he was Assistant Professor at the Communication Systems Division (Commsys) in the Electrical Engineering Department (ISY) at Linkoping University, Sweden. From 2010 to 2011, he was a Postdoctoral Researcher at Commsys. He has previously worked as a Systems and Algorithm Designer in the Wireless Systems Group at Texas Instruments, Bangalore (India) (2003 - 2007). From 2000 to 2003, he worked with Ishoni Networks, Inc., Santa Clara, CA (USA), as a Senior Chip Architecture Engineer. From 1998 to 2000, he was an ASIC Design Engineer with Philips, Inc., Bangalore.

His main research interests include wireless communication using large antenna arrays, coding and signal processing for wireless communication systems, and statistical signal processing. He is a Member of the IEEE, the IEEE Communication Society, the IEEE Signal Processing Society, and the IEEE Information Theory Society. He has been a Technical Program Committee member for several IEEE sponsored conferences (International Conference on Communications (ICC' 2013, 2014), the IEEE Vehicular Technology Conference (VTC) Spring 2013, and the IEEE Swedish Communication Theory Workshop (Swe-CTW) Fall 2012). He has also served as a Guest Editor for a special issue on Massive MIMO in the Journal of Communication Networks (JCN). He also holds two US patents on detection and precoding of Massive MIMO signals. He was awarded the Young Indian Researcher Fellowship by the Italian Ministry of University and Research (MIUR) for the year 2009-10. He was also awarded the CENIIT (Linkoping University) research grant for the year 2012. 